\title{
COMPRESSIVE INSTABILITIES IN METAL-COATED POLYMER MICROTRUSSES
}

\author{
Bharat Bhaga \\ University of Toronto Institute for Aerospace Studies \\ Toronto, Ontario \\ bharat.bhaga@mail.utoronto.ca
}

Abstract-Lightweight structures directly contribute to the sus-
tainability of aviation, as their use reduces the structural weight
of aircraft which in turn reduces fuel burned during flight. One
family of lightweight structures are metal-coated polymers. Hybrid
nanocrystalline microtrusses are a member of this family. These
structures are fabricated by 3D printing complex truss-like structures
out of polymer material, and electrodepositing nanocrystalline metal
onto the polymer. Recent work has shown that buckling instabilities
govern the strength of these systems. Hence this study focuses on
modelling local shell buckling, one of the critical buckling mecha-
nisms. This paper briefly reviews existing models for filled-shell local
shell buckling, and outlines the development of an improved model.
Index Terms-compression; buckling; filled-shell; microtruss;
nanocrystalline; instability

\section{INTRODUCTION}

Electrodepositing nanocrystalline metals onto 3D printed polymers creates hybrid materials with exceptional properties, including high strength and stiffness-to-density as well as nearly limitless geometric control. These hybrid materials can be used to reduce the weight of aircraft and other transportation vehicles, directly decreasing fuel consumption, cost and environmental impact. To achieve this, accurate models of the mechanical behaviour of hybrid nanometal-polymer materials and structures must be developed. This paper will explore the compressive properties of polymer struts enhanced with nanocrystalline metal coatings.

A nanometal polymer hybrid structure is produced by creating a polymer preform through $3 \mathrm{D}$ printing and subsequently coating the preform with nanocrystalline metal [1], as seen in Fig. 1. The reduced grain sizes of nanocrystalline metals relative to conventional metals allow for increased strength as per the Hall-Petch effect [2]. While other properties of nanocrystalline metals, such as wear resistance, also benefit by reducing grain size [3], ductility and modulus tend to decrease. Truss-like structures are very appealing because of their high ratio of stiffness-to-mass. These structures exhibit mass-efficiency and low-weight while combining the highstrength benefits of nanocrystalline metals.

The production methods of nanometal-polymer hybrids also support the goals of sustainability. 3D printing of polymer structures yields less material waste than typical subtractive techniques, while also allowing for the manufacturing of complex geometries with ease. Furthermore, the use of electrodeposition techniques is cost-efficient and is relatively benign environmentally [3]. Elecrotrodeposition also allows for controlling the deposited nanometal crystal sizes [3, 4]. Together

\author{
Craig A. Steeves \\ University of Toronto Institute for Aerospace Studies \\ Toronto, Ontario \\ csteeves@utias.utoronto.ca
}

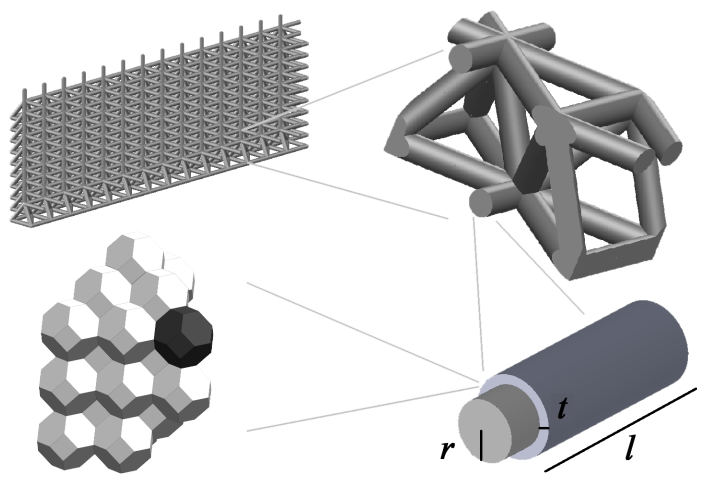

Fig. 1: Various scales of a nanocoated microtruss structure. Clockwise from upper left: coated microtrus structure, truss unit cells, individual struts, atoms at the material level [image modified from [4]]

with control over the geometry of the struts $(r, l$ and $t$ in Fig 1), this provides four length scales over which the behaviour of the nanocoated microtruss structure can be controlled.

Previous studies on nanocoated microtrusses have shown that their failure mechanisms are driven by compressive instabilities of the struts [4]. As the truss elements of nanocoated microtrusses are metal-coated polymer cylinders (as seen in Fig. 1), the present research explores the compressive instabilities associated with metal coated polymer cylinders.

\section{Literature REVIEW}

The two compressive instabilities of interest for metalcoated polymer cylinders are local shell buckling and global (or Euler) buckling, as seen in Fig. 2. Local shell buckling is characterized by the appearance of waves on the surface of the cylindrical shell $[5,6]$, while global buckling results in the lateral deflection of a column relative to its original undeformed axis.

For a nanometal-polymer hybrid, the nanometal coating provides all of the structural strength, and hence failure of the hybrid is governed by failure of the nanometal coating. Past investigations of metal-coated microtrusses assumed that the failure mechanisms of the struts only depended on the geometry and material properties of the metal shell [4], and that the polymer core provided no structural contribution. However, for thinly coated microtruss structures, where the cross-sectional area of the polymer is much more than that of the shell, the polymer contributes to the load-bearing capacity 


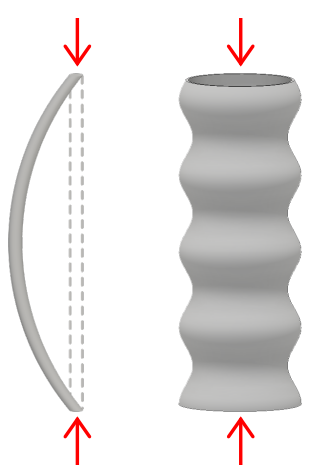

Fig. 2: Appearance of global buckling (left) and local shell buckling (right)

of the struts, while increasing their resistance to buckling [79].

\section{A. Hollow Shell Buckling}

Local shell buckling of filled cylindrical shells - or simply filled-shell buckling - is closely related to local buckling of hollow shells. The local shell buckling load for a thin cylindrical shell in axial compression was found independently [10] by Lorenz [11], Timoshenko [6], and von Karman and Tsien [12]. At the inception of buckling, the strain energy of the shell consists only of the direct compressive stress and strain. Buckling occurs when it is more energy efficient to deform the shell in sinusoidal waves than to continue to deform the shell by direct compression. For a thin cylindrical shell in axial compression, the buckling stress is found to be $[6,11,13-15]$ :

$$
\sigma=\frac{E}{\sqrt{3\left(1-\nu^{2}\right)}} \frac{t}{r}
$$

where $E$ is the Young's modulus of the shell's material, $\nu$ is its Poisson's ratio, and $t$ and $r$ are the thickness and inner radius of the shell, respectively.

Various derivations of (1) have shown that the buckled shape of the shell - be it axisymmetric or non-axisymmetric - is not a factor in determining its buckling load [6, 12]. In addition, the end conditions also do not affect the buckling load provided that the cylinder is sufficiently long $[6,12,13,15]$.

Experimental investigations of hollow-shell buckling have shown that buckling loads in practice are strongly affected by geometric imperfections, material imperfections, and loading asymmetry $[13,16]$. This was confirmed through the experiments of Tennyson [17], who manufactured nearly imperfection-free cylinders and ensured careful axial load distributions around the circumferences of the cylinders.

\section{B. Filled shell buckling}

Filled-shell buckling models utilize separate treatments of the shell and core behaviours. Most models for filled-shell buckling are intended for foam-filled metal or polymer tubes and experiments to validate them have used these material combinations.

The problem of local shell buckling of filled cylindrical shells was driven by the need to understand buckling of solid propellant rockets under axial loads $[7,8,18]$. The earliest attempt at a filled-shell buckling model by Myint [8] emulated the approach used by von Karman and Tsien [12]. Uniquely, the Myint model assumed that a shear interaction between the shell and core was important. Later studies showed that the shear interactions between the shell and the core were negligible [7, 9]. More recently, Karam and Gibson [7] utilized a foundation model derived by Gough et al. [16], which was originally intended for buckling of face sheets on sandwich panels subject to axial compression.

A variety of experimental investigations were also undertaken to investigate the phenomena of filled shell buckling. The majority of the experiments were performed using metal tubes filled with polymer or polymer-like foams [18, 19], intended to mimic the structure of solid propellant rockets or natural materials like plant stems. Through these experiments, it was reported that the filler material prevented or hindered the formation of some buckling deformations. It was also found that a better agreement between theory and experiment occurred with shells when the thickness-to-radius ratios were large [18, 19], due to the fact that buckling loads for thick shells are less prone to imperfection sensitivity.

While the Karam and Gibson model is currently the best available model for filled-shell buckling, there are several drawbacks. The Karam and Gibson model utilizes a stress function originally intended for buckling of face sheets on composite sandwich beams $[16,20]$, and is thus not axisymmetric. Moreover, their model becomes less accurate when the coating thickness is large [20] as the core shear stresses become too large to properly capture using their model.

\section{CORE MOdel}

The development of a core model requires an understanding of the behaviour of a solid polymer cylinder. During local shell buckling of a filled cylinder under an axial load, the core is subject to radial sinusoidal displacements which are the consequence of buckling, as shown in Fig. 3. The following assumptions are utilized:

- the shell and core are perfectly bonded;

- both the shell and core materials behave elastically;

- the buckling displacements are sinusoidal;

- there are many waves that form when the shell buckles;

- the core conforms to the buckled shape of the shell; and

- the shell is very thin compared to the radius (i.e. $t / r<0.1)$.

The Southwell model employs stress functions to calculate the stress field in an axysymmetrically-loaded isotropic cylinder [21]. This describes the stress state of a thick hollow or solid cylinder, depending upon the boundary conditions. Fig. 4 shows the generalized geometry and appropriate boundary conditions for which the Southwell model is valid. The solution to this domain using the Southwell model provides the complete stress state of the axisymmetric section, and hence the entire cylinder. 


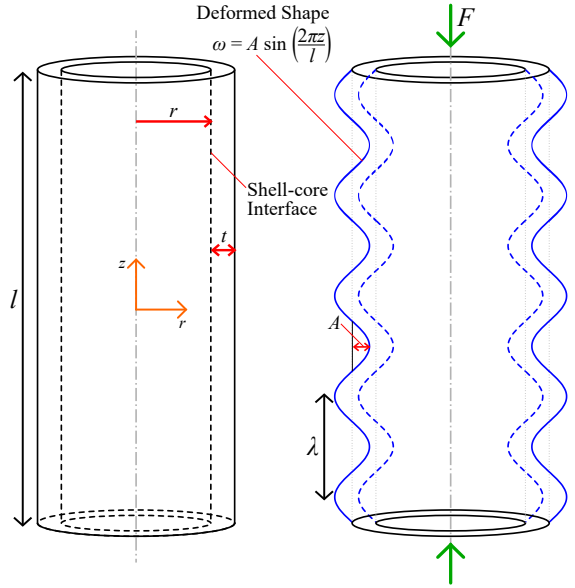

Fig. 3: A metal-coated polymer cylinder prior to buckling deformations (left) and at after buckling (right). The cylinder has radius $r$, length $l$ and shell thickness $t$ while the buckle wavelengths have amplitude $A$ and wavelength $\lambda$ caused by the axial load $F$

While commercial finite element packages are effective in determining the stress state of many geometries quite readily, the use of a custom-written program developed solely for analysing this geometry is a more efficient solution. The solution of the Southwell model is tailored to represent the specific stress states of an axisymmetric domain, allowing the use of coarser meshes to achieve an accurate solution. The governing equations for the Southwell model are [21]:

$$
\begin{gathered}
\frac{\partial^{2} \phi}{\partial r^{2}}-\frac{1}{r} \frac{\partial \phi}{\partial r}+\frac{\partial^{2} \phi}{\partial z^{2}}=0 \\
\frac{\partial^{2} \psi}{\partial r^{2}}-\frac{1}{r} \frac{\partial \psi}{\partial r}+\frac{\partial^{2} \psi}{\partial z^{2}}=\frac{\partial^{2} \phi}{\partial z^{2}},
\end{gathered}
$$

where $r$ is the radial ordinate direction, $z$ is the axial ordinate direction, and $\phi$ and $\psi$ are stress functions which must be determined. Once $\phi$ and $\psi$ are obtained, the individual stress components can be calculated using their equations written in terms of $\phi$ and $\psi[21]$ :

$$
\begin{aligned}
\sigma_{r} & =\frac{1}{r}\left(\frac{\partial \phi}{\partial r}+\frac{\partial \psi}{\partial r}\right)-\frac{1}{r^{2}}[\psi+(1-\nu) \phi] \\
\sigma_{\theta} & =\frac{\nu}{r} \frac{\partial \phi}{\partial r}+\frac{1}{r^{2}}[\psi+(1-\nu) \phi] \\
\sigma_{z} & =-\frac{\nu}{r} \frac{\partial \psi}{\partial r} \\
\tau_{z r} & =\frac{1}{r} \frac{\partial \phi}{\partial z},
\end{aligned}
$$

where $\sigma_{r}$ is the radial stress, $\sigma_{\theta}$ is the circumferential stress, $\sigma_{z}$ is the axial stress and $\tau_{z r}$ is the shear stress as seen in Fig. 4. It is to be noted that the Karam and Gibson model does not account for the circumferential stress [7, 20].

A general analytical solution is not obtainable for (2). A finite differencing solution was developed by Allen et al. [21] which utilizes second-order central differencing throughout the domain. Since the stress components are written in terms of $\phi$ and $\psi$ (i.e. (3)) the governing equations of these stress functions (i.e. (2)) are discretized. Details of the discretization can be found in Allen [21]. With the stress distributions known

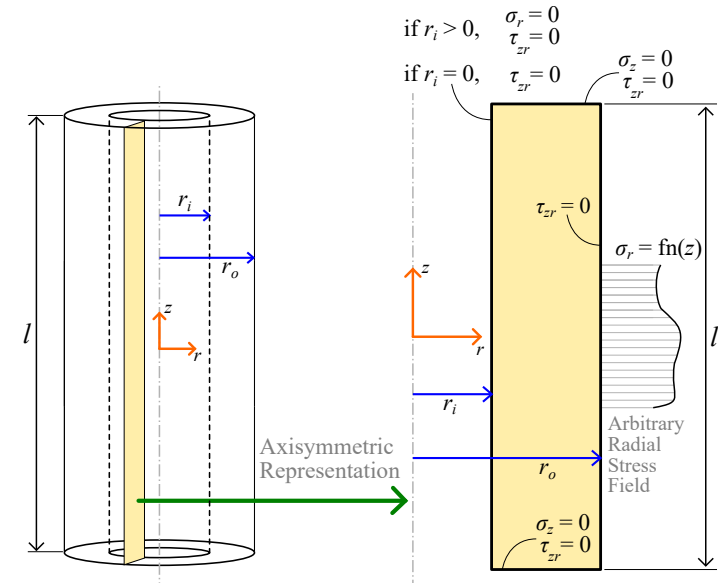

Fig. 4: Cylinder geometry (left) and axisymmetric domain (right) for Southwell model. The cylinder has length $l$, inner radius $r_{i}$ and outer radius $r_{o}$. Boundary conditions for the domain extremeties are given in terms of the values of the shear stress $\tau_{z r}$, axial stress $\sigma_{z}$ and radial stress $\sigma_{r}$

for the axisymmetric domain of Fig. 4, the internal energy of the cylinder can be readily calculated.

A modified domain is used in conjunction with the Southwell model in order to determine the stress state in the core of a filled cylindrical shell undergoing buckling deformations. A sinosoidal stress loading is utilized because buckling deformations are sinusoidal [6-9, 12, 14, 15, 19], and these lead to sinusoidal stress fields. A section of the core undergoing buckling deflections is analysed using the Southwell model, as shown in Fig. 5. Comparing Fig. 4 and Fig. 5, the new domain makes use of periodic boundary conditions, while a sinosoidal radial stress loading acts on the cylinder and the axisymmetric section. The periodic boundary conditions ensure that a calculation done for one buckle wavelength $\lambda$ can be reused when many waves are present $[6,7,12]$. The sinusoidal radial stress field is of the form:

$$
\sigma_{r}=\sigma_{0} \cos \left(\frac{m \pi z}{l}\right)
$$

where $\sigma_{0}$ is a given load magnitude, $m$ is the number of half waves, and $l$ is the length of the cylinder. Since the loading configuration represents one full wavelength, $m$ is set to 2 . The application of the radial stress field (i.e. (4)) leads to sinusoidal radial deflections with a magnitude of $A$ as seen in Fig 3 .

Analyses using the Southwell model, as well as finite element analyses, reveals that for small wavelengths to radius ratios (i.e. small $\lambda / r$ ) the value of the internal energy, $U$, is insensitive to the buckle wavelength $\lambda$. This implies that one calculation using the Southwell model for a given core material will provide the fitting constant $K$ using:

$$
K=\frac{2 U}{m A^{2} \pi r},
$$

where $K$ is the fitting constant. Once $K$ is determined, the expression for the core energy $U_{p}$ is found for any core radius $r$ and number of buckle wavelengths $m / 2$ :

$$
U=U_{p}=\frac{m}{2} K A^{2} \pi r
$$




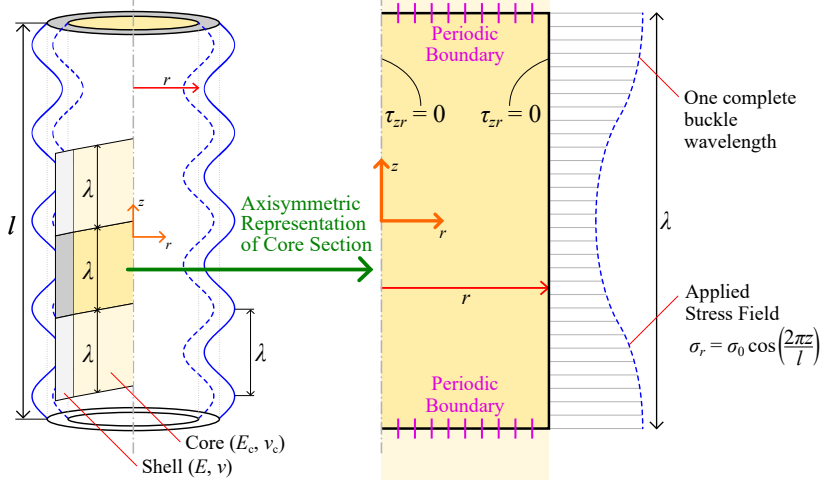

Fig. 5: Geometry, boundary conditions, and load for modified Southwell domain corresponding to buckling deflections of a filled cylindrical shell

This surrogate model is required for the core behaviour so that the core energy can be calculated for various core geometries, while also providing a differentiable function for the internal energy. The value of $K$ is dependant only on the material properties of the core, and must be recalculated when a new core material is used. The core energy expression (Eqn. 6) is used together with the shell energy terms to determine the buckling load for the filled shell system.

\section{BUCKLING LOAD DERIVATION}

While undergoing buckling, the energy sinks of a hollow cylindrical shell include the bending energy $\left(U_{b}\right)$ and the circumferential stretching energy $\left(U_{c}\right)$. The geometry and relevant material properties of a filled shell system are shown in Fig. 3 and Fig. 5.

The shell bending energy refers to the energy associated with the bending action of the shell sections as they undergo sinusoidal buckling deformations. It takes the form [6]:

$$
\begin{aligned}
U_{b} & =\int_{0}^{l}\left[\frac{A^{2} m^{4} \pi^{4}}{l^{2}} \sin ^{2}\left(\frac{m \pi x}{l}\right)\right]\left[\frac{\pi r E t^{3}}{6\left(1-\nu^{2}\right)}\right] \mathrm{d} x \\
& =\frac{\pi E r t^{3}}{24\left(1-\nu^{2}\right)} \frac{m^{4} \pi^{4}}{l^{4}} A^{2} l,
\end{aligned}
$$

where $t$ is the shell thickness, $E$ is the Young's Modulus of the shell material, $l$ is the length of the shell, $r$ is the shell's radius, and $\nu$ is the shell material Poisson's ratio.

The energy resulting from the circumferential tension or compression of the shell hoop sections is defined as [6]:

$$
\begin{aligned}
U_{c} & =\int_{0}^{2 \pi} \int_{r}^{r+t} \int_{0}^{l} \int_{0}^{\epsilon_{r c}} \frac{E}{r} A \sin \left(\frac{m \pi z}{l}\right) r \mathrm{~d} \epsilon \mathrm{d} z \mathrm{~d} r \mathrm{~d} \theta \\
& =\frac{\pi E t A^{2} l}{2 r} .
\end{aligned}
$$

During buckling, the total internal energy $\left(U_{i}\right)$ of a filled cylinder is the sum of the shell bending energy (7), the shell circumferential stretching energy (8), and the core energy (6). The external energy $\left(U_{e}\right)$ arises due to the applied axial force, $F$ (see Fig. 3). These two energy terms, respectively, are:

$$
U_{i}=U_{b}+U_{c}+U_{p}
$$

and

$$
U_{e}=\frac{F m^{2} \pi^{2} A^{2}}{4 l}
$$

At the inception of buckling, the following differential relation holds:

$$
\frac{\partial U_{e}}{\partial A}=\frac{\partial U_{i}}{\partial A}
$$

This is known as the bifurcation point: a further increase in $F$ will either lead to buckling or continued axial loading without buckling. While in perfect systems buckling will not take place, imperfections will always cause real systems to buckle [13].

Equating (9) and (10) and applying the operation as in (11), the elastic buckling load of a metal-coated polymer cylinder in axial compression is found. Adding on the load-bearing capacity of the core gives an expression of the form:

$$
F=\left(1+\frac{E_{c}}{E} \frac{r}{2 t}\right)\left[\frac{2 \pi^{3} r E t^{3}}{3\left(1-\nu^{2}\right)} \frac{1}{\lambda^{2}}+\frac{E t}{2 r \pi} \lambda^{2}+\frac{K r}{\pi} \lambda\right] \text {. }
$$

Equation (12) applies for any $\lambda$, but only one critical $\lambda$ value exists for a given cylinder with known material properties. This critical $\lambda$ value is found by finding the positive real root of the polynomial expression $\partial F / \partial \lambda=0$. Once found, the critical $\lambda$ value is substituted into (12) to give the critical load for the filled shell system of interest.

\section{Finite Element Verification}

Buckling analysis was undertaken in Abaqus to verify the predicted loads. This was done for ranges of cylinder lengthto-radius $(l / r)$ ratios and thickness-to-radius $(t / r)$ ratios. Many $l / r$ ratios were used to ensure that the buckling load was insensitive to cylinder length. All geometries were represented axisymmetrically, and the subspace algorithm was used to solve for the eigenvalues and corresponding buckled shapes. A perturbation was applied in the form of an axial displacement at one end of each cylinder, while keeping the opposing end fixed and allowing both ends to expand radially. There were at least 5 elements through the coating thicknesses after meshing was carried out. The lowest eigenvalue calculated by the solver was taken to be the buckling load.

The $t / r$ ratios studied ranged between 0.005 and 0.1 . Ratios below 0.005 indicate very low thicknesses which are not practical for nanocoated microtruss structures, while those above 0.1 correspond to very thick coatings which are outside of the range of thin-shell buckling assumptions.

Fig. 6 shows a graph of the non-dimensional buckling loads as predicted by Abaqus along with the theoretical buckling loads predicted by both the new elastic theory (i.e. (12)) and the Karam and Gibson theory [7]. The theoretical curves and Abaqus results were found for $E_{c} / E=0.01$, this value being typical for metal-coated polymer cylinders where the polymer material is ABS or a similar 3D printed material. The buckling loads for different $l / r$ values (overlapping points at each $t / r$ value in Fig. 6) are not identical due to slight differences in mesh characteristics. However, the are sufficiently close 


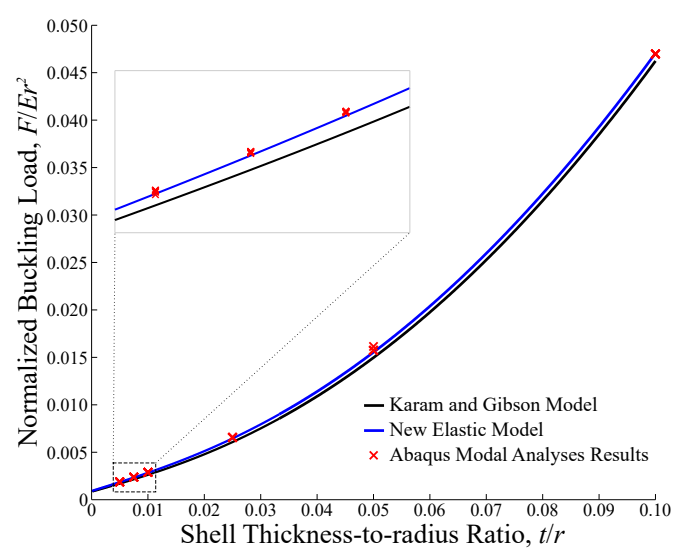

Fig. 6: Non-dimensional loads calculated for the new elastic model and the Karam and Gibson model, with Abaqus modal analyses results included $\left(E_{c} / E=0.01, \nu=0.3\right.$ and $\left.\nu_{c}=0.35\right)$

to indicate no dependence of the buckling load on cylinder length.

As seen in Fig. 6, both the Karam and Gibson theory and the new elastic theory predict lower loads than the Abaqus results. The Karam and Gibson theory predicts loads that are up to $10 \%$ lower than those predicted by Abaqus, while the new theory predicts loads that are no less than $3 \%$ of the values. The agreement between the Abaqus results and the new model theory improves at higher $t / r$ values.

\section{A. Discussion}

The improved predictions of the new elastic model are in accordance with the increased energy that the this model predicts. The circumferential stress component found using the Southwell model is not accounted for in the Karam and Gibson model, leading to lower energy predictions and lower predicted buckling loads. While the Southwell model is a more complete description of the stress state of a core subject to buckling deformations, the Karam and Gibson theory is simpler to implement in practice.

Though the Southwell model involves more steps to find the behaviour of the elastic core (i.e. finding the fitting, $K$ ), the loading configuration can be changed for several other situations involving filled shell buckling. This can include hollowing out the core or assuming no adhesion between the shell and the core. These cases have not been studied in the existing literature.

While the present model predicts the buckling strength of metal-coated polymer cylinders, adapting it for use with hybrid microtruss structures will require topological information of a given microtruss.

\section{CONCLUding REMARKS}

A new model has been developed that accurately accounts for the energy state in the polymer core of a metal-coated polymer cylinder as the cylinder undergoes sinusoidal buckling deformations. The model utilizes the Southwell stress model, which describes the stress state in cylindrical geometries under axisymmetric loads. This model has been used to represent the core behaviour of a filled-shell system undergoing buckling, and agrees more closely with finite element results. Although the model is more complex to implement than existing theories, such as the Karam and Gibson method, it is more adaptable to different cylinder geometries or adverse shellcore adhesion situations.

\section{REFERENCES}

[1] L. Gordon, B. Bouwhuis, M. Suralvo, J. Mccrea, G. Palumbo, and G. Hibbard, "Micro-truss nanocrystalline Ni hybrids," Acta Materialia, vol. 57, no. 3, pp. 932-939, Feb. 2009.

[2] K. Kumar, H. Van Swygenhoven, and S. Suresh, "Mechanical behavior of nanocrystalline metals and alloys," Acta Materialia, vol. 51, no. 19, pp. 5743-5774, Nov. 2003.

[3] U. Erb, "Electrodeposited Nanocrystals: Synthesis, Properties and Industrial Applications," Nanostructured Materials, vol. 6, no. 5-8, pp. 533-538, 1995

[4] A. Lausic, C. Steeves, and G. Hibbard, "Effect of grain size on the optimal architecture of electrodeposited metal/polymer microtrusses," Journal of Sandwich Structures and Materials, vol. 16, no. 3, pp. 251$271,2014$.

[5] K. R. F. Andrews, G. L. England, and E. Ghani, "Classification of the Axial Collapse of Cylindrical Tubes under Quasi-Static Loading," International Journal of Mechanical Sciences, vol. 25, no. 9, pp. 687696, 1983.

[6] S. P. Timoshenko and J. M. Gere, Theory of Elastic Stability. New York: McGraw-Hill, 1961.

[7] G. N. Karam and L. J. Gibson, "Elastic Buckling of Cylindrical Shells with Elastic Cores - Part I: Analysis," International Journal of Solids and Structures, vol. 32, no. 8/9, pp. 1259-1283, 1995.

[8] T. Myint-U, "Stability of Axially Compressed Core-Filled Cylinders," AIAA Journal, vol. 4, no. 3, pp. 552-553, 1965.

[9] P. Seide, "The Stability Under Axial Compression and Lateral Pressure of Circuluar-Cylindrical Shells with a Soft Elastic Core," Journal of the Aeronautical Sciences, vol. 29, no. 7, pp. 851-862, 1962.

[10] J. Teng and J. Rotter, Buckling of Thin Metal Shells. London: CRC Press, 2004.

[11] R. Lorenz, "Achsensymmetrische verzerrungen in dunnwandingen hohlzzylindern," Zeitschrift des Vereines Deutscher Ingenieure, vol. 52, no. 43, pp. 1706-1713, 1908

[12] T. von Karman and H.-S. Tsien, "The Buckling of Thin Cylindrical Shells under Axial Compression," Journal of the Aeronautical Sciences, vol. 8, no. 8, pp. 303-312, 1941.

[13] D. Bushnell, "Buckling of Shells - Pitfall for Designers," AIAA Journal, vol. 19 , no. 9, pp. 1183-1226, Sep. 1981.

[14] L. Donnell, "A New Theory for the Buckling of Thin Cylinders Under Axial Com pression and Bending," Transactions of the ASME, Aeronautical Engineering, vol. AER-56-12, pp. 795-806, 1935.

[15] S. B. Batdorf, "A Simplified Method of Elastic Stability Analysis for Thin Cylindrical Shells: Part I - Donnell's Equation,” NACA TN 1341, 1947.

[16] G. Gough, C. Elam, and N. de Bruyne, "The Stabilization of a Thin Sheet by a Continuous Supporting Medium," Journal of the Royal Aeronautical Society, vol. 44, pp. 12-43, 1940.

[17] R. Tennyson, "An Experimental Investigation of the Buckling of Circular Cylindrical Shells in Axial Compression using the Photoelastic Technique," UTIAS Report No. 102, 1964.

[18] G. N. Karam and L. J. Gibson, "Elastic Buckling of Cylindrical Shells with Elastic Cores - Part 2: Experiments," International Journal of Solids and Structures, vol. 32, no. 8/9, pp. 1285-1306, 1995.

[19] W. S. Goree and W. A. Nash, "Elastic Stability of Circular Cylindrical Shells Stabilized by a Soft Elastic Core," Experimental Mechanics, vol. 2, no. 5, pp. 142-149, 1962

[20] H. G. Allen, Analysis and Design of Structural Sandwich Panels. Hungary: Pergamon Press, 1969.

[21] D. d. G. Allen, Relaxation Methods in Engineering and Science. New York: McGraw-Hill, 1954. 